\title{
Multiscale Theory of Dislocation Climb
}

\author{
Pierre-Antoine Geslin, ${ }^{*}$ Benoît Appolaire, and Alphonse Finel \\ Laboratoire d'Etude des Microstructures, Onera/CNRS, 29, avenue de la division Leclerc, 92322 Châtillon, France \\ (Received 16 June 2015; revised manuscript received 21 October 2015; published 23 December 2015)
}

\begin{abstract}
Dislocation climb is a ubiquitous mechanism playing a major role in the plastic deformation of crystals at high temperature. We propose a multiscale approach to model quantitatively this mechanism at mesoscopic length and time scales. First, we analyze climb at a nanoscopic scale and derive an analytical expression of the climb rate of a jogged dislocation. Next, we deduce from this expression the activation energy of the process, bringing valuable insights to experimental studies. Finally, we show how to rigorously upscale the climb rate to a mesoscopic phase-field model of dislocation climb. This upscaling procedure opens the way to large scale simulations where climb processes are quantitatively reproduced even though the mesoscopic length scale of the simulation is orders of magnitude larger than the atomic one.
\end{abstract}

DOI: 10.1103/PhysRevLett.115.265501

PACS numbers: $61.72 . \mathrm{Bb}, 46.35 .+\mathrm{z}, 62.20 . \mathrm{Hg}$

Complex multiscale phenomena are ubiquitous in materials science and make the prediction of macroscopic properties a formidable challenge. In metallic alloys, macroscopic properties are often controlled by the interplay between different phases or precipitates and the nucleation, multiplication, and propagation of defects such as dislocations, cracks, voids, etc. These phenomena are driven by atomic forces that manifest themselves at mesoscopic time and length scales, out of reach of atomistic simulation techniques. A solution to this dilemma is to develop mesoscopic coarse-grained models that operate at intermediate time and length scales between the atomic and the macroscopic scales we want to reach. Obviously, an accurate mesoscopic description must incorporate lower scale fluctuations and, therefore, a proper choice of the intermediate scales is such that the degrees of freedom that operate at lower scale reach a quasiequilibrium state at the mesoscale. These degrees of freedom will then be embedded into mesoscopic parameters. This kind of multiscale approach has been successfully developed to study quantitatively alloy solidification $[1,2]$ and nucleation of new phases [3] at mesoscopic scales.

Plastic deformation in crystals results from the movement of dislocations along specific crystallographic planes. At high temperatures, when vacancy diffusion is activated, dislocations change glide plane by absorbing or emitting vacancies. Therefore, this climb mechanism plays a central role in the plastic deformation of metals. In this Letter, we propose an upscaling procedure for dislocation climb proceeding in two steps. First, we analyze the lower scale problem of the interaction between dislocations and vacancies. We derive an analytical expression for the climb rate of a dislocation presenting a periodic distribution of jogs. Attachment-detachment kinetics of vacancies to the dislocation core is taken into account, as well as pipe diffusion between jogs. Therefore, our model goes beyond the usual approaches of climb, where the exchange kinetics is always neglected and where the presence of jogs and core diffusion are either ignored [4-7] or at best approximately treated [8]. Next, we deduce the activation energy of the climb process from the analytical climb rate, bringing new insight into previous experimental measurements. Finally, we present a phase field model (PFM) that treats dislocations at the mesoscale. We show how to link rigorously the kinetic parameter of the PFM to the lower scale analysis.

Climb rate of a jogged dislocation.-Climb processes do not operate homogeneously along the dislocation line, but rather at specific defects, called jogs, distributed along the line [see Fig. 1(a)]. The formation energy of jogs is often high, in particular in face-centered cubic (fcc) metals, and their density may be small. In addition to this simple argument, experimental observations [9-13] suggest that climb might be limited by low jog concentrations. Therefore, a realistic description of climb must account for bulk diffusion of vacancies toward the dislocation core, the exchange dynamics between bulk and core, diffusion along the core between the jogs, and finally attachmentdetachment kinetics at the jogs. As testified by atomistic calculations [14-16], these mechanisms are controlled by various energy barriers depicted in Fig. 1(b): the bulk and

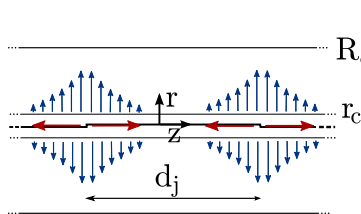

(a)

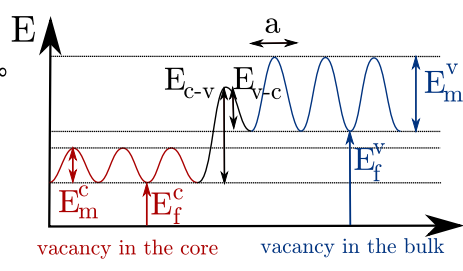

(b)
FIG. 1 (color online). (a) Schematic vacancy diffusion around a jogged dislocation. Red and blue arrows illustrate, respectively, core and bulk vacancy diffusion. (b) Energy profile experienced by a vacancy diffusing in the core (red) and escaping to the volume (blue). 
core vacancy formation (respectively, migration) energies $E_{f}^{v}$ and $E_{f}^{c}$ (respectively, $E_{m}^{v}$ and $E_{m}^{c}$ ), the exchange energy barriers $E_{v-c}$ and $E_{c-v}$ from the bulk to the core and vice versa. The vacancy diffusion coefficients in the bulk and in the core are, respectively, defined as $D_{v}=D_{0} \exp \left(-\beta E_{m}^{v}\right)$ and $D_{c}=D_{0} \exp \left(-\beta E_{m}^{c}\right)(k T=1 / \beta$ denotes the thermal energy), where $D_{0}$ is related to the Debye frenquency $\nu$ and the interatomic distance $a$.

We consider a straight edge dislocation of core radius $r_{c}$ with a regular distribution of jogs separated by a distance $d_{j}$ [see Fig. 1(a)]. As commonly assumed [13,17], we consider that the influence of the jog velocity on the diffusion profiles is negligible, and we obtain two steady-state diffusion equations associated, respectively, to the bulk and core regions. For simplicity reasons, the attachmentdetachment kinetics at the jogs is assumed to be instantaneous compared to other processes [18]. Therefore, in the immediate neighborhood of the jogs, vacancies are at thermodynamic equilibrium where the osmotic force, proportional to the chemical potential of vacancies, balances the climb component of the Peach-Koehler force acting on the dislocation and generated by the external stress $\sigma^{a}$. The vacancy concentration at $r=R_{\infty}$ is considered to be maintained at $c_{\infty}$, representing a possible supersaturation or under saturation of vacancies far from the dislocation.

By generalizing the procedure of Gilmer et al. [19] to our cylindrical geometry, we show in the Supplemental Material [20] that the diffusion equations can be exactly solved for the bulk and core vacancy fields, and therefore for the vacancy fluxes. The exact climb rate expression contains an infinite sum of terms involving modified Bessel functions. However, a straightforward but still precise approximation (we comment on that point in Fig. 2) leads to the following simple expression:

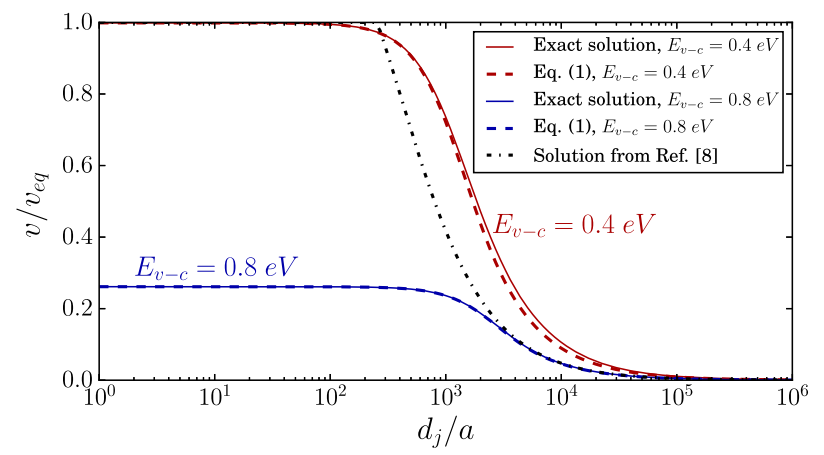

FIG. 2 (color online). Climb rate $v$ in units of $v_{\text {eq }}$ of Eq. (5) (see text) as a function of the interjog distance for two volume-core exchange energy barriers. In red: $E_{v-c}=0.4 \mathrm{eV}$ [full line: exact expression, dashed line, Eq. (1)]; in blue: $E_{v-c}=0.8 \mathrm{eV}$ [Eq. (1) and exact expressions are indistinguishable]. In dashed black: climb rate according to Ref. [8].

$$
v=\frac{\frac{2 \pi D_{v} c_{v}^{0}}{b}\left(\frac{c_{\infty}}{c_{v}^{0}}-e^{\beta \sigma^{a} \Omega}\right)}{\ln \left(\frac{R_{\infty}}{r_{c}}\right)+\frac{l_{v}^{2}}{r_{c}^{2}}\left[1+2 \alpha^{2}\left(\frac{d_{j}}{2 \alpha l_{c}} \operatorname{coth} \frac{d_{j}}{2 \alpha l_{c}}-1\right)\right]},
$$

where $c_{v}^{0}=\exp \left(-\beta E_{f}^{v}\right)$ is the equilibrium vacancy concentration in the bulk, $\Omega$ is the atomic volume, and where the characteristic length scales $l_{c}$ and $l_{v}$ and the coefficient $\alpha$ are defined by

$$
\begin{aligned}
l_{c}^{2} & =\frac{D_{c} r_{c}}{a \nu} \exp \left(\beta E_{c-v}\right), \\
l_{v}^{2} & =\frac{D_{v} r_{c}}{a \nu} \exp \left(\beta E_{v-c}\right), \\
\alpha^{2} & =\frac{l_{v}^{2}+r_{c}^{2} \ln \left(R_{\infty} / r_{c}\right)}{2 l_{v}^{2}} .
\end{aligned}
$$

The length scale $l_{c}$ is the typical diffusion length of a vacancy in the dislocation core, whereas $l_{v}$ is the diffusion length associated to the attachment time to the dislocation core: if $l_{v} \ll r_{c}$, a vacancy coming close to the core will be absorbed before it diffuses away. We now present in Fig. 2 an analysis of the climb rate given in Eq. (1). We set the temperature to $T=600 \mathrm{~K}$ and use material parameters pertaining to aluminum [21-23] $\left(E_{m}^{v}=0.61 \mathrm{eV}, E_{m}^{c}=\right.$ $0.35 \mathrm{eV}, \quad E_{f}^{v}=0.67 \mathrm{eV}, \quad E_{f}^{c}=0.50 \mathrm{eV}, \quad D_{0}=$ $1.51 \times 10^{-5} \mathrm{~m}^{2} / \mathrm{s}, \quad \nu=9.3 \times 10^{13} \mathrm{~s}^{-1}, \quad a=0.285 \mathrm{~nm}$, $\left.r_{c}=1 \mathrm{~nm}\right)$. The results are presented in the form $v / v_{\mathrm{eq}}$, where

$$
v_{\mathrm{eq}}=\frac{2 \pi D_{v} c_{v}^{0}}{b \ln \left(R_{\infty} / r_{c}\right)}\left(\frac{c_{\infty}}{c_{v}^{0}}-e^{\beta \sigma^{a} \Omega}\right)
$$

is the climb rate obtained when the dislocation line is supposed to be an homogeneous and perfect sink or source of vacancies, i.e., at local equilibrium with vacancies [4]. This is the climb rate used in the most recent analysis where climb has been incorporated in dislocation dynamics codes [5-7,24,25] and in dislocation density based theories [26]. When the volume-core exchange barrier is small (typically $\left.E_{v-c}<E_{m}^{v}\right), v / v_{\text {eq }}$ is close to 1 only if the interjog distance $d_{j}$ is small enough. In that case, attachment kinetics to the core and jog density are high enough for the vacancies to reach a local equilibrium all along the dislocation line and, therefore, the climb process is simply controlled by bulk diffusion. However, when $d_{j}$ increases beyond a critical threshold which depends on core diffusion, the climb rate decreases significantly and the local equilibrium hypothesis is no longer valid. When the attachment kinetics is slow $\left(E_{v-c}>E_{m}^{v}\right), v / v_{\mathrm{eq}}$ is always significantly smaller than 1. The climb process is now mostly controlled by the attachment kinetics and, for large $d_{j}$, also by core diffusion. The expression proposed in [8] (dashed line in Fig. 2) does not incorporate the significant influence of the attachment 
kinetics nor the overlap of the diffusion profile from neighboring jogs.

Activation energy.-The dislocation climb rate is often difficult to extract precisely from experimental observations partly because deformation often occurs through other mechanisms such as dislocation glide. Nonetheless, because dislocation climb is the limiting process, the activation energy of creep measured experimentally can be associated to the climb process alone. In our theoretical approach, the apparent activation energy of dislocation climb can be computed as $E_{a}=-\partial \ln \left(\left.v\right|_{d_{j}, f_{m}}\right) / \partial \beta$. We consider here that the jog population is constant and maintained out of equilibrium through nonthermally activated mechanisms such as dislocation intersections [4]. Also, the effect of the driving force $f_{m}=c_{\infty} / c_{0}-$ $\exp \left(\beta \sigma^{a} \Omega\right)$ is withdrawn for generality reasons by computing the activation energy at $f_{m}$ constant. This effective activation energy is plotted in Fig. 3 in the case of slow $\left(E_{v-c}=0.8 \mathrm{eV}\right)$ and fast $\left(E_{v-c}=0.4 \mathrm{eV}\right)$ volume-core exchanges. In the latter case and for small interjog distances, the activation energy $E_{a}$ converges to the selfdiffusion activation energy $E_{f}^{v}+E_{m}^{v}$ as expected in the case of high jog concentration [4]. On the other hand, for larger interjog distances, $E_{a}$ drops to smaller values. In this regime, the core diffusion becomes a limiting process and the activation energy converges to an intermediate value between the bulk self-diffusion and the core selfdiffusion energy $E_{f}^{c}+E_{m}^{c}$. It is interesting to note that the drop of climb rate is necessary accompanied by the activation energy dropping to lower values than the selfdiffusion energy.

In Ref. [27], Sherby et al. reported that the creep activation energy varies from the self-diffusion energy to a plateau value of $1.19 \mathrm{eV}$ when the creep temperature decreases. This value is significantly lower than the selfdiffusion energy of aluminum and the authors have pointed out the disagreement between their results and the creep models existing at the time. In light of our results, the drop of the activation energy could be interpreted as a decrease

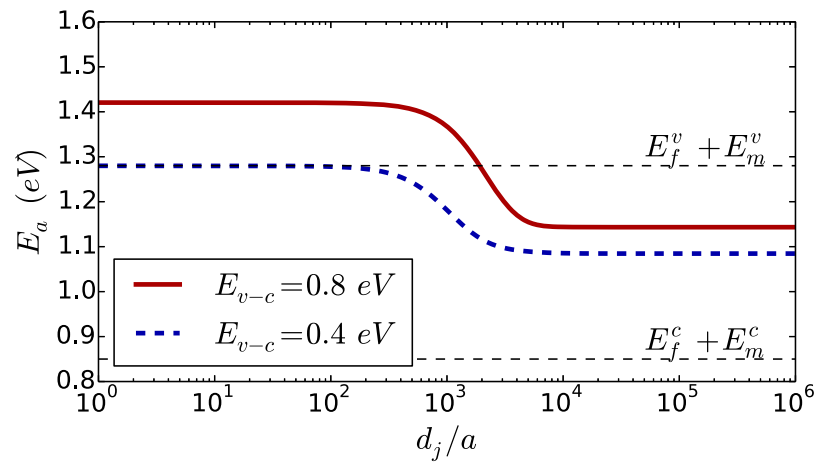

FIG. 3 (color online). Apparent activation energy vs interjog distance for different temperatures and for $E_{v-c}=0.4 \mathrm{eV}$ and $E_{v-c}=0.8 \mathrm{eV}$. of the jog density along the dislocation line at lower temperatures. Albeit this interpretation is appealing, it must be kept in mind that these experiments were performed on polycrystalline samples where grain boundary diffusion could be another deformation mechanism at low temperatures.

Figure 3 also shows that for slow volume-core exchanges $\left(E_{v-c}=0.8 \mathrm{eV}\right)$ and at high jog concentrations, the volume-core exchange process becomes limiting and the activation energy is larger than the self-diffusion energy.

This can be related to the creep experiments performed by Edelin et al. on magnesium samples [11-13]. The authors show that the activation energy associated to dislocation climb is approximately $1.8 \mathrm{eV}$, significantly greater than the self-diffusion energy of $\mathrm{Mg}: 1.43 \mathrm{eV}$. They try to interpret this result by considering that the jog density is close to thermal equilibrium if the jog formation energy is $E_{j}=1.8-1.43=0.37 \mathrm{eV}$. However, such a low jog density leads to climb rates several orders of magnitude lower than reported [13]. Moreover, the micrographs in Ref. [11] show that dislocations are curved in their climb plane, implying a high jog density. Another interpretation of these experimental measurements can be made by considering that the unexpectedly elevated activation energy is representative of the energy barrier for volume-core exchanges. In the high jog density regime, such an energy barrier leads to an apparent activation energy higher than the one of self-diffusion (Fig. 3), while the climb rate is only moderately decreased (Fig. 2).

Upscaling to a phase field model (PFM).-The main motivation of the Letter is to upscale the atomic scale dislocation properties to a larger mesoscopic scale. The PFM is one of the most popular methods to handle complex microstructure and interface evolutions at mesoscale. Recently, it has been extended to incorporate dislocation climb $[28,29]$. We show here how to inform this PFM with the above description of the climb of jogged dislocation. The PFM relies on two fields: the vacancy concentration field $c$ and a plastic field $\phi$. A dislocation loop is identified as the boundary between a platelet of thickness $d$, within which $\phi(r)=1$, and the matrix within which $\phi(r)=0$. The free energy is written as

$$
\begin{aligned}
F= & \int d r^{3}\left\{\frac{1}{2 \beta \Omega c_{v}^{0}}\left(c-c_{v}^{0}\right)^{2}+A \phi^{2}(1-\phi)^{2}+\frac{B}{2}|\boldsymbol{n} \wedge \boldsymbol{\nabla} \phi|^{2}\right. \\
& \left.+\frac{1}{2}\left[\epsilon_{i j}-\epsilon_{i j}^{0}(c, \phi)\right] C_{i j k l}\left[\epsilon_{k l}-\epsilon_{k l}^{0}(c, \phi)\right]-\sigma_{i j}^{a} \epsilon_{i j}\right\}
\end{aligned}
$$

The first contribution is the vacancy chemical free energy approximated by its second order expansion around the bulk equilibrium concentration $c_{v}^{0}$. The second and third contributions represent the dislocation core energy; $\boldsymbol{n}$ is a unit vector perpendicular to the vacancy loop and coefficients $A$ and $B$ control the core properties, in particular its width $w=\sqrt{2 B / A}$. The last two components represent the 
elastic energy: $C_{i j k l}$ are the elastic constants, $\sigma_{i j}^{a}$ the applied stress, and $\epsilon_{i j}^{0}(c, \phi)=[h(\phi) / 2 d]\left(b_{i} n_{j}+b_{j} n_{i}\right)+\delta_{i j}\left(V^{*} / 3 \Omega\right) c$ the eigenstrain tensor associated with plastic and vacancy fields; $h(\phi)$ is a monotonic function such that $h(0)=0$, $h(1)=1$, and $h^{\prime}(0)=h^{\prime}(1)=0$ and $V^{*}$ is the vacancy relaxation volume. Considering the marginal effect of elastic field generated by the vacancies $[29,30]$, we neglect this contribution and set $V^{*}=0$.

We turn now to the field evolution equations. The dislocation loops shrink and grow by exchanging vacancies with the bulk in such a way that the combined field $\psi=$ $c+c^{*} \phi$ is conserved $\left(c^{*}=b / d\right.$ is the number of vacancies involved in a unitary climb process). Therefore, $\psi$ obeys a Cahn-Hilliard equation, $\dot{\psi}=\nabla \cdot M \nabla(\delta F / \delta \psi)$, whereas the plastic field $\phi$ follows a simple Allen-Cahn equation, $\dot{\phi}=-L(\delta F / \delta \phi)$. Using the more natural fields $c$ and $\phi$, we obtain

$$
\begin{aligned}
& \dot{c}=\nabla \cdot M \nabla \frac{\delta F}{\delta c}+L c^{*}\left(\frac{\delta F}{\delta \phi}-c^{*} \frac{\delta F}{\delta c}\right), \\
& \dot{\phi}=-L\left(\frac{\delta F}{\delta \phi}-c^{*} \frac{\delta F}{\delta c}\right) .
\end{aligned}
$$

Because elastic relaxation is much faster than diffusive processes, the elastic fields $\varepsilon_{i j}$ are supposed to relax quasistatically. Accordingly, we impose elastic equilibrium by setting $\left(\partial \sigma_{i j} / \partial x_{j}\right)=0$ where the local stress is defined by $\sigma_{i j}=\left(\delta F / \delta \varepsilon_{i j}\right)+\sigma_{i j}^{a}$. It appears in Eq. (7) that the coefficient $M$ is simply the vacancy mobility which, in the limit of small concentration, is given by $M=\beta \Omega D_{v} c_{v}^{0}$ [31]. The mesoscopic mobility coefficient $L$ controls the exchanges between the fields $\{c\}$ and $\{\phi\}$, i.e., the rate of absorption or emission of vacancies at the dislocation core. Thus, it can incorporate phenomenologically the lower scale behavior of jogs and core diffusion discussed above. For the purpose of upscaling these properties, we perform an asymptotic analysis of the PFM and derive a closed-form expression for the climb velocity. We consider a dislocation dipole introduced with a platelet (where $\phi=1$ ) of thickness $d$ oriented along the $x$ axis (see inset of Fig. 4). We assume the dipole to be long enough to neglect the interdislocation interactions. The dislocation core width $w$ is chosen to be much smaller than the diffusion length and we consider that the vacancy field is quasistatic. Within these limits, the phase field will be of the form $\phi(x, t)=f\left[x-x_{0}(t)\right]$ and its spatial derivative along the climb direction is sharply peaked around the position $x_{0}(t)$ of the dislocation. Multiplying Eq. (8) by this derivative and integrating in a small domain around the dislocation core leads to a simple equation that relates the climb rate to the concentration $c_{d}$ in the vicinity of the core and to the stress component $\sigma^{a}$ along the direction perpendicular to the vacancy loop:

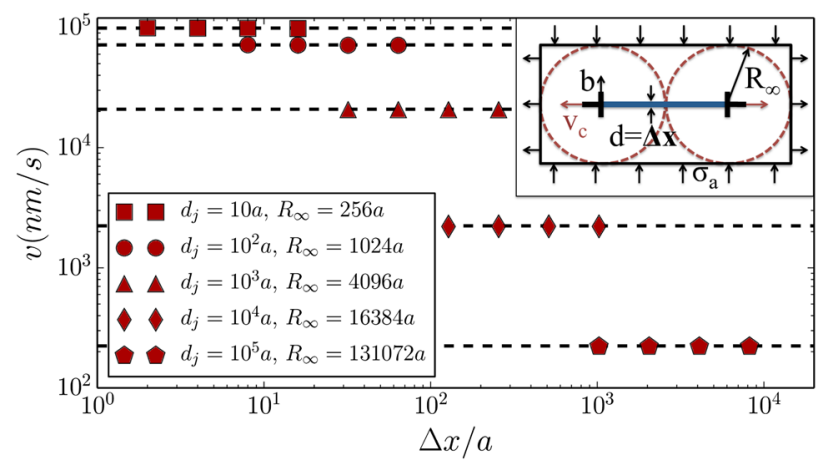

FIG. 4 (color online). Climb rate of an isolated dislocation for different grid spacings $\Delta x$ and interjog distances $d_{j}$. The PFM and exact results are represented by symbols and dotted lines, respectively. The inset shows the configuration used in these simulations.

$$
v_{c}=\frac{3 w b}{d} L\left[\frac{1}{\beta \Omega c_{v}^{0}}\left(c_{d}-c_{v}^{0}\right)-\sigma^{a}\right] .
$$

Within the quasistatic approximation and away from the dislocation core, Eq. (7) reduces simply to $\nabla^{2} c=0$ whose solution in cylindrical symmetry is straightforward. Considering the boundary conditions $c_{\infty}$ at $R_{\infty}$ and an inner boundary condition at $r_{c}^{\text {eff }} \sim w$ where the vacancy concentration reaches $c_{d}$, a simple circular integration of the vacancy flux leads to the following climb rate

$$
v_{c}=\frac{2 \pi D_{v} c_{v}^{0}}{b \ln \left(R_{\infty} / r_{c}^{\mathrm{eff}}\right)}\left(\frac{c_{\infty}}{c_{v}^{0}}-\frac{c_{d}}{c_{v}^{0}}\right) .
$$

Solving (9) and (10) for $c_{d}$ leads to the following PFM climb rate:

$$
v_{\mathrm{PFM}}=\frac{\frac{2 \pi D_{v} c_{v}^{0}}{b}\left(\frac{c_{\infty}}{c_{v}^{0}}-\beta \sigma^{a} \Omega-1\right)}{\ln \left(\frac{R_{\infty}}{r_{c}^{\mathrm{etf}}}\right)+\frac{2 \pi d}{3 w} \frac{\beta \Omega D_{v} c_{v}^{0}}{L b^{2}}} .
$$

By direct identification with Eq. (1) (considering the Taylor expansion of the exponential, valid for the low stresses used in creep experiments), we deduce a link between the PFM parameters (mobility $L$, dislocation core width $w$, and vacancy platelet thickness $d$ ) and the atomic scale dislocation properties (interjog distance $d_{j}$, diffusion length scales $l_{v}$ and $l_{c}$ ):

$$
L=\frac{2 \pi d \Omega D_{v} c_{v}^{0} \beta / 3 w b^{2}}{\ln \left(\frac{r_{c}^{\mathrm{eff}}}{r_{c}}\right)+\frac{l_{v}^{2}}{r_{c}^{2}}\left\{1+2 \alpha^{2}\left[\frac{d_{j}}{2 \alpha l_{c}} \operatorname{coth}\left(\frac{d_{j}}{2 \alpha l_{c}}\right)-1\right]\right\}} .
$$

Thus, the parameter $L$ can be chosen to reproduce quantitatively the climb rate of a jogged dislocation. The other major outcome of this upscaling procedure is that it can be used for any vacancy loop thickness $d$ which, from the numerical point of view, is simply equal to the grid spacing $\Delta x$ used in 
the implementation of the PFM equations. Therefore, any grid spacing $\Delta x$ may be used, provided that the mobility coefficient $L$ is chosen according to Eq. (12). This is illustrated in Fig. 4, where we show that the PFM climb rate is almost identical to the exact one (the difference is lower than $1 \%$ ), even if $\Delta x$ (and therefore the mesoscopic dislocation core width $w$ ) is orders of magnitude larger than the interatomic distance. Thus, this multiscale procedure opens the possibility to study quantitatively the climb behavior of an assembly of dislocations in large scale systems.

We believe that this multiscale procedure can also be readily applied to other physically relevant situations where the interface mobility is affected by its atomic structure such as epitaxial growth of vicinal surfaces or solidification in the case of atomically faceted interface [32].

*pageslin@gmail.com

[1] A. Karma and W.-J. Rappel, Phys. Rev. E 57, 4323 (1998).

[2] A. Karma, Phys. Rev. Lett. 87, 115701 (2001).

[3] Q. Bronchart, Y. Le Bouar, and A. Finel, Phys. Rev. Lett. 100, 015702 (2008).

[4] J. Hirth and J. Lothe, Theory of Dislocations, edited by M. B. Bever, M. E. Shank, C. A. Wert, and R. F. Mehl (Krieger Publishing Company, 1968).

[5] D. Mordehai, E. Clouet, M. Fivel, and M. Verdier, Philos. Mag. 88, 899 (2008).

[6] D. Mordehai, E. Clouet, M. Fivel, and M. Verdier, IOP Conf. Ser.: Mater. Sci. Eng. 3, 012001 (2009).

[7] S. M. Keralavarma, T. Cagin, A. Arsenlis, and A. A. Benzerga, Phys. Rev. Lett. 109, 265504 (2012).

[8] R. Balluffi, Phys. Status Solidi B 31, 443 (1969).

[9] D. N. Seidman and R. W. Balluffi, Phys. Status Solidi B 17, 531 (1966).

[10] A. Sutton and R. Balluffi, Interfaces in Crystalline Materials (Oxford University Press, Oxford, 1995).

[11] G. Edelin and J. Poirier, Philos. Mag. 28, 1203 (1973).

[12] G. Edelin and J. Poirier, Philos. Mag. 28, 1211 (1973).

[13] D. Caillard and J. Martin, Thermally Activated Mechanisms in Crystal Plasticity, edited by R. Cahn, Pergamon Materials Series (Elsevier, Cambridge, UK, 2003) pp. 281-317.
[14] E. Clouet, Acta Mater. 54, 3543 (2006).

[15] T. Lau, X. Lin, S. Yip, and K. Vanvliet, Scr. Mater. 60, 399 (2009).

[16] M. Kabir, T. Lau, D. Rodney, S. Yip, and K. Van Vliet, Phys. Rev. Lett. 105, 095501 (2010).

[17] R. Balluffi and D. Seidman, J. Appl. Phys. 36, 2708 (1965).

[18] In the case of pure fcc metals, molecular dynamics simulations show that no barrier is associated with the absorption or emission of vacancies at a jog [E. Clouet (private communication)].

[19] G. Gilmer, R. Ghez, and N. Cabrera, J. Cryst. Growth 8, 79 (1971).

[20] See Supplemental Material at http://link.aps.org/ supplemental/10.1103/PhysRevLett.115.265501 for a detailed derivation of the climb rate of a jogged dislocation.

[21] G. Purja Pun and Y. Mishin, Acta Mater. 57, 5531 (2009).

[22] T. Volin, K. Lie, and R. Balluffi, Acta Metall. 19, 263 (1971).

[23] Landolt-Bornstein: Numerical Data and Functional Relationship in Science and Technology, edited by $\mathrm{O}$. Madelung (Springer-Verlag, Berlin, 1990), Vol. 25.

[24] B. Bakó, E. Clouet, L. M. Dupuy, and M. Blétry, Philos. Mag. 91, 3173 (2011).

[25] F. Boioli, P. Carrez, P. Cordier, B. Devincre, and M. Marquille, Phys. Rev. B 92, 014115 (2015).

[26] M. Geers, M. Cottura, B. Appolaire, E. Busso, S. Forest, and A. Villani, J. Mech. Phys. Solids 70, 136 (2014).

[27] O. Sherby, J. Lytton, and J. Dorn, Acta Metall. 5, 219 (1957).

[28] P.-A. Geslin, B. Appolaire, and A. Finel, Appl. Phys. Lett. 104, 011903 (2014).

[29] J. Ke, A. Boyne, Y. Wang, and C. Kao, Acta Mater. 79, 396 (2014).

[30] I. Margvelashvili and Z. Saralidze, Sov. Phys. Solid State 15, 1774 (1974).

[31] The expression of the mobility is chosen in accordance with the quadratic approximation of the free energy and enables to obtain a simple Fickean diffusion $\dot{c}=D \nabla^{2} c$ far from the dislocation cores.

[32] J. Dantzig and M. Rappaz, Solidification (EPFL Press, Lausanne, 2009). 